\title{
Reply to: Sarcopenia definition in patients with NAFLD
}

\author{
Yuya Seko ${ }^{1}$
}

Published online: 28 July 2018

(c) Japanese Society of Gastroenterology 2018

\section{To Dr. Tao-Chun Peng}

We appreciate your impressive comment to our manuscript. We would like to answer some questions.

First, Dr. Tao-Chun Peng mentioned about the definition of sarcopenia which differs from the previous studies by Koo et al. and Lee et al. We also estimated the prevalence of sarcopenia by appendicular skeletal muscle mass. However, the significant association between these indices and histological findings was not detected. As we mentioned in the manuscript, the possible reason for this discrepancy was mainly based on size of population in this study. In addition, the lack of hand grip strength was also a limitation of this study.

Second, we totally agree with your opinion that the increased SF ratio contributed to the improvement of liver function. The most important finding in this study was that SF ratio, not only BMI, should be the monitoring marker in NAFLD treatment.

This reply refers to the article available at https://doi.org/10.1007/ s00535-018-1496-5.

Yuya Seko

yuyaseko@koto.kpu-m.ac.jp

1 Department of Gastroenterology and Hepatology, Kyoto

Prefectural University of Medicine, 465 Kajii-cho,

Kawaramachi-Hirokoji, Kamigyo-ku, Kyoto 602-8566, Japan 